\title{
LINGÜÍSTICA
}





\section{¿HALLAR LA AGUJA EN EL ALFILETERO? ENSEÑAR UNIDADES FRASEOLÓGICAS A TRAVÉS DE POESÍA MODERNA}

Nils Bernstein

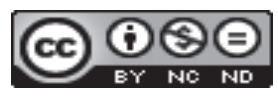

Esta obra está bajo una licencia Creative Commons Reconocimiento-No Comercial-Sin Obra Derivada 



\title{
¿HALLAR LA AGUJA EN EL ALFILETERO? ENSEÑAR UNIDADES FRASEOLÓGICAS A TRAVÉS DE POESÍA MODERNA
}

\author{
FINDING A NEEDLE IN A STICK OF NEEDLES? TEACHING \\ PHRASEOLOGICAL UNITS WITH MODERN POETRY
}

\section{Nils Bernstein}

\begin{abstract}
RESUMEN
El lenguaje según Guillermo Cabrera Infante (Cabrera Infante, 1967, p. 221) es un bosque de palabras. De acuerdo con las ideas del mismo escritor cubano en su novela Tres tristes tigres: A veces usar una Unidad Fraseológica (UF) no es tan difícil como hallar una aguja en el pajar que son en comparación fáciles de hallar sino como hallar la aguja en el alfiletero. Corpas Pastor (2003, p. 109) destaca lacónicamente: "[L]a fraseología [...] está de moda". Y se puede afirmar que sobre todo en la adquisición de segundas lenguas están de moda. Por lo tanto, en este artículo quisiera describir la importancia de estas unidades y mostrar una opción de implementar su enseñanza en la didáctica de lenguas extranjeras. En un primer paso, se brinda un breve resumen de las ventajas de la literatura en la clase de lengua extranjera. Después, se describen los desafíos de la fraseología para los alumnos. Posteriormente se darán ejemplos prácticos. Los autores con los que se va a trabajar son el poeta chileno Nicanor Parra (1914-2018) y el poeta experimental austriaco Ernst Jandl (1925-2000). Al final, a manera de conclusión, haré referencia a dos proyectos: el proyecto del óptimo fraseológico y el proyecto de los modismos más extendidos Widespread Idioms in Europe and Beyond.

Palabras clave: fraseología, literatura en la enseñanza, óptimo fraseológico, Ernst Jandl, Nicanor Parra.
\end{abstract}

\begin{abstract}
According to Guillermo Cabrera Infante (Cabrera Infante, 1967, p. 221) language is a forest of words. The Cuban author also stated in his novel Tres tristes tigres: Sometimes using the right phraseological unit is not as difficult as finding a needle in a haystack, which would be comparatively easy, but rather as difficult as finding a needle in a stack of needles. Corpas Pastor (2003, p. 109) simply asserts: "[L]a fraseología [...] está de moda". And still today phraseology is relevant especially in the didactics of foreign languages. In a first step, we will illustrate benefits of incorporating literature in language classes in general. Afterwards we show these benefits of using the example of the Chilean poet Nicanor Parra (1914-2018) and the Austrian writer of experimental poetry Ernst Jandl (19252000). Concluding with a synthesis, two projects are introduced: the minimum of phraseological units that are to be learned and the project of Widespread Idioms in Europe and Beyond.

Key words: phraseology, literature in second language adquisition, phraseological minimum, Ernst Jandl, Nicanor Parra.
\end{abstract}

Dr. Nils Bernstein. Coordinador de certificados de alemán. Centro de Idiomas, Universidad de Hamburgo. Alemania. Correo electrónico: nils.bernstein@uni-hamburg.de

Recepción: 06- 11- 2017

Aceptación: 14- 01- 2018 


\section{Las ventajas y posibilidades de la literatura en el salón de clase}

Muchos aspectos de la lengua se pueden enseñar a través de literatura en el salón de clase: los dialectos, los estilos elaborados tanto como los restringidos o informales, los temas de la cultura, etc. También se puede emplear la literatura para practicar la fonética y la entonación; o para centrarse en la gramática. De hecho, en todos los niveles del aprendizaje es adecuado el uso de la literatura, incluso desde el inicio del proceso de la adquisición de una segunda lengua. Un posible género literario es la Poesía Concreta cuyos inicios se dieron en el siglo pasado. Este género surgió en varios países del mundo y es considerado por esa razón un fenómeno internacional de poesía. Los textos literarios son auténticos y aumentan la motivación del estudiante. Dada su autenticidad, la recepción resulta ser a veces intrincada.

La tipología de ejercicios con textos literarios es amplia. En la secuencia de enseñanza se puede diferenciar entre ejercicios antes, durante y después de la lectura. Antes de la lectura siempre es de importancia activar el conocimiento previo de los estudiantes, por ejemplo, a través de una hipótesis acerca del contenido de un texto que presupone el título del mismo. Durante la lectura hay varios estilos para la comprensión de lectura, la lectura detallada, selectiva o global. Después de la lectura, hay muchas opciones para trabajar con el texto leído: adivinar el título, escribir títulos para cada párrafo, escribir nuevos textos como una carta al escritor/al protagonista, continuar la historia, escribir una reseña o un texto que responda a la pregunta: ¿Qué habría pasado en caso de que...? Asimismo, en el trabajo del texto literario en la clase de lenguas extranjeras podemos enfocarnos en el léxico, como es el caso de la fraseología.

\section{Los desafíos de la fraseología en la clase de lenguas extranjeras}

Para contestar la pregunta de por qué son complicadas las Unidades Fraseológicas (UFs) en la adquisición de una lengua extranjera, primero debemos tener una definición de lo que se entiende por fraseología. De acuerdo con Corpas Pastor (1998, p. 167), la UF se define como una "combinación estable de unidades léxicas formada por al menos dos palabras gráficas, cuyo límite superior se sitúa en el nivel de la oración compuesta". Por lo tanto, una UF tiene los criterios imprescindibles de (1) la polilexicalidad, (2) la idiomaticidad y la (3) la estabilidad o fijación. Los dos últimos criterios son graduales. (1) El primer criterio es el más fácil de explicar: una UF contiene más que una palabra. La fórmula rutinaria muchas gracias es un ejemplo de ello. Fórmulas como esta son importantes para principiantes de una lengua extranjera y las empleamos en textos muy sencillos. (2) El segundo criterio, la idiomaticidad fue, sobre todo en los años 70 y 80 del siglo pasado, un aspecto muy llamativo para la investigación lingüística. La idiomaticidad significa que no podemos entender una composición léxica en el sentido literal, sino que esta posee un sentido figurado. En el caso de tocar violín, expresión que se usa en Perú y que aparece varias veces en las novelas de Mario Vargas Llosa (cf. Segura García, 1997), queremos decir que una persona es redundante o innecesaria. En alemán, por ejemplo, usamos en el mismo contexto la expresión das fünfte Rad am Wagen sein, "ser la quinta rueda del coche", expresión que tampoco se puede ni entender ni traducir literalmente. Para decir lo que en México significa dejar a alguien frito en Colombia se dice mandar a chupar gladiolos. En vez de estar cultivando margaritas en alemán se dice die Radieschen von unten ansehen, lo que, traducido palabra por palabra, significa: "mirar los rabanitos desde abajo". Esas expresiones tienen una notable incompatibilidad semántica. Los lexemas no corresponden, por lo que los estudiantes posiblemente tienden a consultar un diccionario para 
eliminar sus dudas. (3) La estabilidad o fijación de las unidades también hacen difícil usar las UFs adecuadamente. Puedo decir estimados señoras y señores, pero sería extraño decir *estimados señores y señoras y cambiar los elementos en el eje sintagmático. De igual forma sería extraño cambiarlos en el eje paradigmático *estimados damas y caballeros. En la lengua materna se reproducen de memoria UFs - que están compuestas por más de una palabra- de igual manera como se reproducen palabras aisladas. Pero no funciona de esa manera en una segunda lengua donde se tiene que aprender y reproducir palabra por palabra y, por lo tanto, necesita una capacidad de memoria más grande. Tenemos que memorizar todos los elementos de la UF y reproducirlos en el orden exacto. Por lo general, el aprendizaje de UFs sigue los cuatro pasos que diferencia Lüger (1997, p. 102) y que constituyen la metodología más adecuada para la enseñanza de UFs en el salón de clase:

1. Reconocer

2. Descifrar

3. Aprender

4. Usar, aplicar

Según los criterios mencionados, contamos en el rubro de la fraseología con paremias (De tal palo tal astilla), con fórmulas rutinarias (muchas gracias, hasta luego), con colocaciones (gracias a, entender por, politicamente correcto), con frases hechas y citas famosas que pueden ser de origen literario (En algún lugar de la Mancha de cuyo nombre no quiero acordarme) o de origen político o histórico (Yes, we can! -refrán de Barack Obama que quizás nadie recordará en unas décadas y actualmente modificado por Angela Merkel con su frase Wir schaffen das!). Como se ha puesto de relieve, los criterios característicos de las UFs complican su uso correcto por parte de los estudiantes, no obstante, las UFs forman parte del léxico en todos los niveles de una lengua extranjera.

\section{El apoyo de la literatura para la enseñanza de UFs. Ejemplos}

\subsection{Observaciones acerca de UFs en la literatura}

En la publicación Sobre las funciones de los fraseologismos en textos literarios Zuluaga propone el siguiente sistema de diferenciación. Las UFs son realmente usadas en su "función fraseológica“ (Zuluaga, 2006, p. 631), con una "connotación o evocación” (2006, p. 633), en su "función icónica" (íbid., p. 634) o finalmente en su "función lúdico-poética" (íbid., p. 637). Todas esas funciones son funciones textuales mientras la desautomatización (íbid., p. 637) es con pleno derecho un criterio típico para textos literarios y en la misma se puede observar los efectos de "ambigüedades, intertextualidad, reflexión metalingüística, refuerzo de la percepción y de la atención, orientación hacia las formas mismas, humor” (íbid., p. 637).

En el caso de las UFs idiomáticas se puede constatar una cierta desviación del lenguaje que puede resultar - dependiendo del contexto- en una desautomatización. Las UFs idiomáticas cumplen con la función poética que describió Roman Jakobson (1979, p. 92). Dicha función se pone de manifiesto cuando la construcción lingüística elegida intenta producir un efecto especial en el destinatario, por ejemplo, goce, emoción o entusiasmo. Sin embargo, usamos UFs con una función conativa porque transportamos un mensaje al destinatario de acuerdo con la teoría de los actos de habla que describió John Robert Searle. Por su afinidad con la función poética y su inherente grado de poeticidad, la fraseología evoca un cierto interés en escritores y poetas que tienden a modificar las UFs con varias técnicas. 


\section{a. Ernst Jandl (1925-2000), poeta austríaco}

El poeta Ernst Jandl es mencionado en muchas publicaciones por su conexión con la Poesía Concreta, una moda y técnica internacional de la poesía experimental, aunque no toda su obra es Poesía Concreta sino solamente una parte de ella. En los libros de alemán como lengua extranjera sus poemas aparecen muy a menudo y, por lo general, esos eran los poemas más exitosos (p. ej., Ottos Mops, fünfter sein, schtzngrmm, wien: heldenplatz). Jandl tiene varias técnicas de juegos de lenguaje. Una es la modificación y la desautomatización de UFs. En el poema que no es experimental, Pharmakologisch, pone un refrán de Johann Wolfgang von Goethe (1749-1832) cuya obra Jandl conocía muy bien por su carrera y puesto de profesor de alemán e inglés en un colegio estatal. En el original de Goethe, el poema Canción del arpista que aparece en el capítulo 13 del segundo libro de la novela Los años de aprendizaje de Wilhelm Meister (1795/96), podemos encontrar la cita famosa: "Quien nunca en llanto el pan mojó/ [...] ese os ignora, potencias del cielo" (Wer nie sein Brot mit Tränen aß,/ [...] der kennt euch nicht, ihr himmlischen Mächte). Se usa esa cita también para decir que uno necesita cierta experiencia para hablar sobre asuntos complicados de la vida. Como en el caso de muchos refranes, también se hizo famosa la parodia del refrán de Goethe, p. ej.: "Quien nunca en llanto el pan mojó, no sabe como pican las migajas" (Wer nie sein Brot mit Tränen aß, weiß nicht wie Krümel pieksen). La parodia de Jandl en Pharmakologisch es: "Brot mit Tränen gegessen/ ist würzig und nahrhaft,/ es beruhigt die Nerven, es/ macht satt” (1997, Tomo I, p. 60) (Mojé pan en llanto/ es bien condimentado y nutritivo/ calma los nervios, nos/ llena; traducción propia). Mientras el arpista en la novela de Goethe de hecho llora cantando esos versos, vemos en esa función lúdico-poética, como la describe Zuluaga, que una UF causa un efecto cómico al ponerla en un contexto que permite interpretarla literalmente. Algo parecido ocurre en el poema experimental Hörprobe (Ensayo auditivo o Soundcheck) (Jandl, 1997, Tomo 3, p. 37). Es muy difícil traducir el poema y transportar su significado, así como las alusiones, por lo tanto, agrego una versión traducida palabra por palabra y una que transporta las alusiones, lo que debe de expresar según las asonancias.

Versión alemana:

höherhören [...]

höhere hören

und daumen

höhere daumen

und hören

höhere hören

und höhere daumen

meine höheren daumen

meine höheren hören

kennen sie mich herren

kennen sie mich herren

kennen sie mich herren

meine damen und herren (Jandl, 1997, Tomo 3, p. 37)

Versión literal:

Escuchar más alto [...]

distinguidos escuchan

y dedos pulgares

distinguidos dedos pulgares

y escuchan 


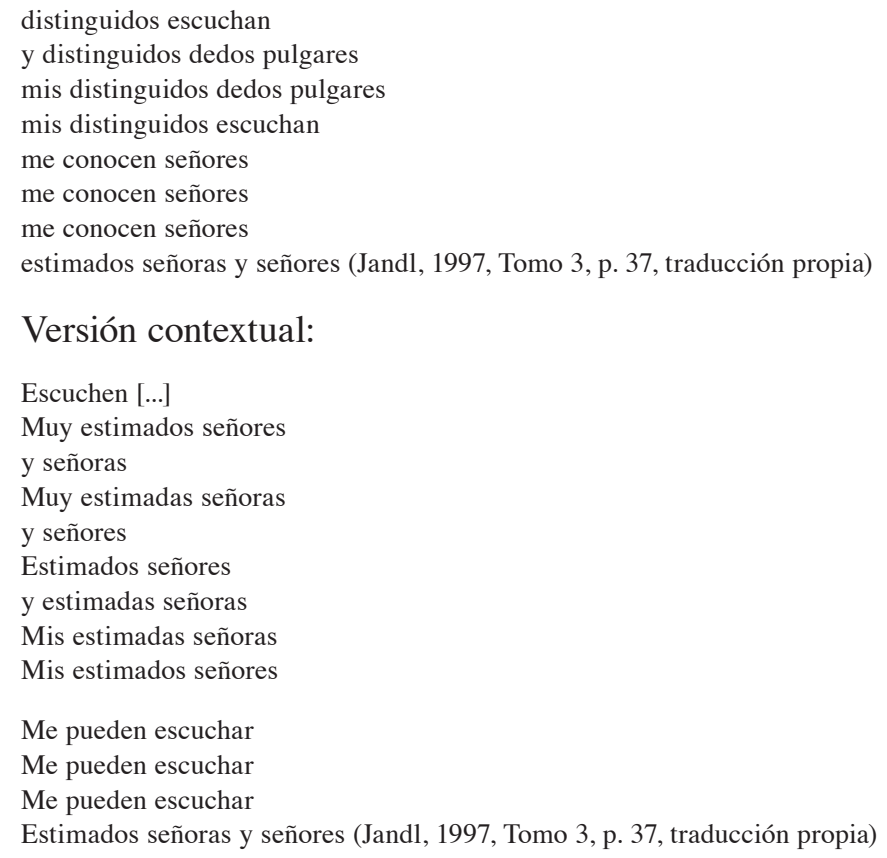

Tanto en la versión literal como en la contextual aparece la UF sin modificación, en su forma original. Aparentemente ese Soundcheck no funcionó adecuadamente. Solo al final queda un detalle claro mientras que todo lo articulado anteriormente por el yo poético es un disparate. El ejemplo nos demuestra que sí se puede leer este poema en una clase de principiantes. Además, deja ver que existen UFs muy importantes en un estilo menos elevado y menos complejo en el proceso de aprendizaje de una lengua extranjera.

\section{b. Nicanor Parra (1914-2018), poeta chileno}

Nicanor Parra, galardonado con el Premio Cervantes en 2011, es conocido por su colección Poemas y antipoemas (1954). Desde aquel entonces fue nombrado "antipoeta" por la crítica literaria, un apodo que el propio poeta aplica y acepta. El nombre es conocido por su origen de la obra del poeta chileno Vicente Huidobro (1893-1948) que escribió en el verso 282 del Canto IV de su obra Altazor o el viaje en paracaídas de 1931: "Aquí yace Vicente antipoeta y mago" (Huidobro, 2010, p. 64). Es empíricamente justificado tomar a Nicanor Parra como un ejemplo de poeta con afición a la fraseología por antonomasia. En Poemas y antipoemas encontramos en 13 de 29 poemas una o más UFs, la mayoría en la tercera parte de la colección. La densidad de UFs que podemos calcular por la división, poemas divididos entre poemas con UFs, es entonces de 0,45 UFs por poema. En la colección Hojas de Parra (1985) -cuyo título también es una UF- encontramos 19 poemas con UFs entre 67 poemas en total y el resultado de la densidad de UFs con 0,28 aún es alta. Sin embargo, la variedad del castellano, el chileno, no es un "idiomatic language" (Brennan, 2007, p. 10) -ninguna lengua como tal es idiomática sino solamente contiene elementos idiomáticos. A pesar de la alta densidad de UFs en la obra de Parra, no se puede afirmar que el poeta usa UFs sistemáticamente. Es consciente de su uso, como destaca en una entrevista hecha el 5 de noviembre de 2009 en Las Cruces, en la cual declara no manejar una determinada técnica para implementar UFs en sus poemas. Tiene una debilidad por frases hechas, pero al mismo tiempo cuestiona su alta frecuencia y la falta de consciencia en el 
uso de la gente común y corriente. Además de esa crítica del lenguaje como herramienta adecuada para dibujar los pensamientos, yuxtapone el sentido idiomático con el sentido literal para causar un efecto cómico. Los ejemplos más convincentes y más obvios son sus Obras Públicas de 2006 y los Trabajos Prácticos de 1996 donde yuxtapone la UF con un objeto expuesto, p. ej. un huevo junto con el texto "El huevo de Colón" o el mismo objeto con la frase hecha "El descubrimiento de América" (Parra, 2006b, sin paginación). Muy famoso además es el texto "Naturaleza muerta" el cual aparece al lado de un jitomate atravesado por un clavo.

Los poemas de Nicanor Parra también son aptos para usarse en niveles para principiantes pero, dependiendo de la formulación de los ejercicios, se pueden usar en niveles más avanzados. Muy famoso es el poema Pido que se levante la sesión en los Versos de Salón (1962):

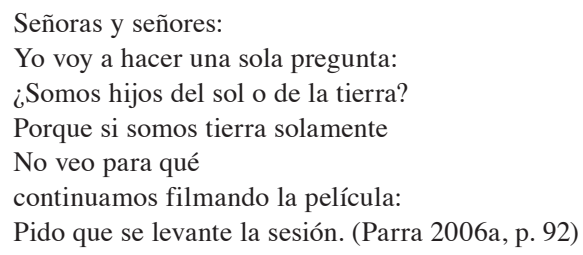

Nótense las UFs Señoras y señores en el primer verso y la de pedir que + Verbo subjuntivo + Sustantivo en el último. Preguntas y ejercicios para introducir ese poema al salón de clase pueden ser: ¿Qué significa Señoras y señores y en qué contexto pragmático se usa esa fórmula rutinaria? ¿Es adecuado usar una fórmula rutinaria de esa especie en un poema? ¿Por qué o por qué no? ¿En qué contexto pide una persona "levantar una sesión”? ¿Qué podría significar "levantar una sesión" en el contexto del poema? ¿Qué podría significar "hijos del sol o de la tierra" en el tercer verso? Se nota que el estilo del poema no es muy elaborado, no hacen falta muchos conocimientos de lengua para entender el sentido literal. No obstante, con las preguntas formuladas se puede explotar su potencial para provocar una discusión profunda acerca de las UFs.

En la obra de Parra encontramos una gran cantidad de UFs enlistadas a continuación en su forma original y no tal y como las modifica el poeta: estar cayendo a pedazos (Parra 2006a, p. 33), morirse de rabia (p. 35), señoras y señores (p. 41 y 92), séptimo sentido (ibíd.), arrancarse los cabellos (p.45), último centavo, desvanecimientos prematuros, caridad pública, estar muerto de hambre (p. 49), reír a carcajadas, romperse la cabeza, el lecho de muerte, meterse en camisa de once varas (p. 89), sacar a alguien de quicio (p. 104), poner fin a algo (p. 108), estar harto de (p. 113), pequeño burgués, tragar saliva (p. 115), correr como sangre (p. 119), una sed ardiente de, dar la paliza a algo (p. 23), carne de gallina (p. 128), dar diente por diente, falta de tacto (p. 129), acometer molinos de viento (p. 130), pactar con el diablo, caja de Pandora (p. 132), construir castillos en el aire (p. 144), levantar la copa (p. 153), hoja de laurel (p. 155), poco a poco (p. 160), muerto de rabia (p. 164), se prohíbe + infinitivo (p. 179), silla de ruedas (p. 182), con mis propios ojos (p. 183), Padre nuestro que estás en el cielo (p. 185), Cordero de Dios (p. 186), diestra y siniestra (p. 189), contante y sonante (p. 192), hasta luego (p. 195), guardar rencor (p. 200), no tener arte ni parte (p. 214), un lobo disfrazado de cordero (p. 231), nunca perdió más tiempo el águila que cuando escuchó los consejos del cuervo (p. 236), romper los moldes (p. 237), zutano mengano fulano, sic transit gloria mundi (p. 256), en un abrir y cerrar de ojos (p. 274), comulgar con ruedas de molino (p. 280), hablar hasta por los codos (p. 303), todos los caminos conducen a Roma (p. 304), canto de cisne (p. 306), Dios lo tenga en su santo reino (p. 310), a buen entendedor pocas palabras bastan (p. 311), poner 
algo/a alguien en tela de juicio (p. 334), a boca cerrada no entran moscas (p. 348), estar con la soga al cuello (p. 379), más vale tarde que nunca (p. 455), el pueblo unido, jamás será vencido (p. 461), el reverso de la medalla (p. 710).

\subsection{Ejemplos didácticos para el salón de la clase}

Implementamos a continuación un ejemplo poético con sugerencias didácticas tanto de Ernst Jandl como de Nicanor Parra. El poema de Jandl es el ya mencionado Hörprobe. La construcción sigue una conmutación de vocales, sobre todo de 'e' y la metafonía 'ö', además de 'a' y del diptongo 'au'. El cambio no es absolutamente consecuente. Si fuera consecuente tendría que cambiar la ‘ö’ por una 'e' también en el verso 11 en la palabra höhere que evita el poeta. Sin embargo todas las palabras que son modificadas existen en el léxico alemán -un método que no siempre se aplica en la poesía experimental si rememoramos palabras como "Kroklokwafzi” en el famoso poema Das große Lalula (1932) de Christian Morgenstern, o "the borogroves" en el poema no menos famoso Jabberwocky (1871) de Lewis Carroll. Jandl prefiere generar palabras existentes que no obstante en su orden sintagmático no tienen sentido. Los alumnos/as podrían cambiar en un primer paso lúdico las vocales para producir un texto que sí se pudiese entender pero que perdería el valor poético. Además, en una lectura del texto logrado se puede modificar la entonación como la usa el poeta mismo. En la primera estrofa Jandl no implementa ninguna entonación ni modulación, mientras que en la segunda y tercera estrofa cambia la voz (Jandl, 1969/1971/2001, track 22). Sin mostrar el título del poema se pueden hacer las siguientes preguntas en el salón de clase:

- ¿Cuál podría ser el título del texto?

- ¿Cuáles son las vocales que son cambiadas?

- ¿Cuál sería la versión "correcta” de ese texto?

- ¿En qué situaciones se usan las formas rutinarias "höhere Herren und Daumen" y "meine Damen und Herren"?

Por otro lado, el ejemplo de Nicanor Parra es una fotografía con un título o texto agregado por el poeta que publicó y mostró en la exposición de sus así llamadas Obras Públicas. ${ }^{1}$

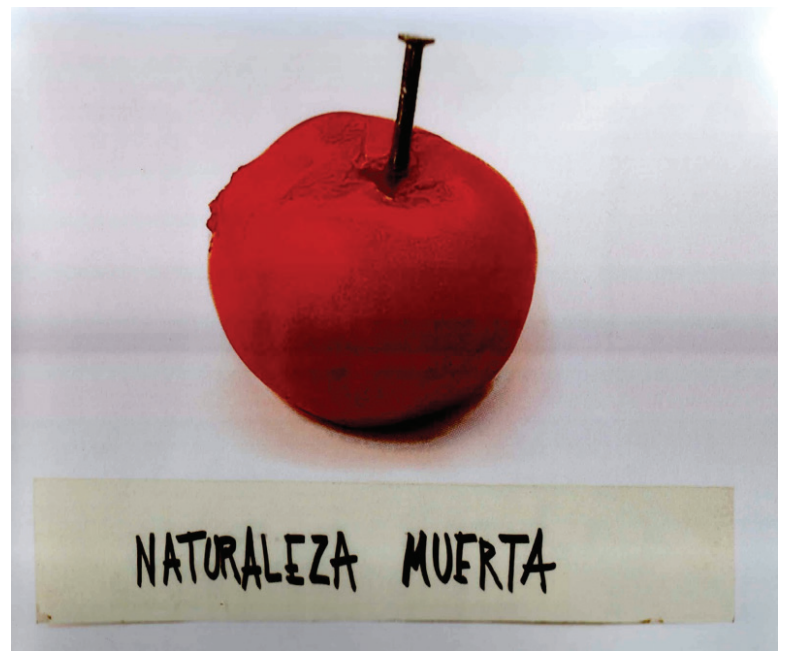

Figura 1. "Naturaleza muerta" de Nicanor Parra (Parra, 1996, sin paginación) 
Este trabajo de un poeta no es un poema clásico sino más bien algo parecido a un "objet trouvé" en el sentido de Marcel Duchamp. Además se lo puede entender como una parodía de bodegones clásicos de la tradición artística europea con su punto culminante en el siglo XVII. La UF forma parte del título y del texto y es representada en su significado literal en el dibujo: un jitomate que es perforado por un clavo y, por lo tanto, aparentemente entregó su alma -acto que en la exposición en el Palacio de la Moneda fue realizado doblemente porque el objeto no fue cambiado durante toda la exposición y en consecuencia enmoheció y perdió su color rojo para ganar un color negro y verde. El término naturaleza muerta con su sentido literal está actualizado a través del contexto. No se lo puede traducir a cualquier lengua; en alemán será "Stillleben" casi como en inglés "still-life". La palabra francesa "nature mort" e italiana "natura morta" es parecida a la versión castellana. En el salón de clase se pueden aplicar las siguientes preguntas/sugerencias de trabajo:

- ¿Podrían describir el dibujo?

- ¿Cuál podría ser un título adecuado? (Dado que el título está escondido). O al revés: ¿Cuál podría ser el dibujo adecuado al título? (Dado que el dibujo está escondido).

- ¿Cómo se dice naturaleza muerta en su lengua materna?

- ¿Cuáles serían ideas parecidas a la del dibujo?

Una hoja de trabajo, modificado de Lüger (1997, p. 125f), facilita el contexto de unidades fraseológicos y garantiza el aprendizaje del léxico.

Tabla 1. Hoja de trabajo

\begin{tabular}{|l|l|}
\hline Forma elemental: & Clase de unidad fraseológica: - \\
\hline & \\
\hline Variantes & Restricciones morfosintácticas \\
\hline & \\
\hline Contexto & Condiciones del uso: ¿Quién, cuándo, a quién? \\
\hline & \\
\hline Significado, paráfrasis & Correspondencias en la lengua materna, circunscripción \\
\hline & \\
\hline Hiperónimo, expresiones afines & Contexto histórico, etimología \\
\hline & \\
\hline
\end{tabular}

\section{El proyecto "Widespread Idioms" y el proyecto del "óptimo fraseológico"}

Una ayuda muy relevante en la cuestión acerca de los modismos más importantes es el proyecto de los "Widespread Idioms", los modismos extendidos "in Europe and beyond" que encabezó Elisabeth Piirainen (Piirainen, 2012). Como "Widespread Idioms", modismos extendidos, entendemos unidades que tienen un núcleo léxico y significado idiomático muy semejante o casi igual y que se usan en varias lenguas (Piirainen, 2010, p. 167). Un "Widespread Idiom" muy común es por ejemplo to swim against the tide/stream que se puede traducir palabra por palabra al español como nadar contra corriente. El proyecto tiene, desde su inicio en el año 
2004, el apoyo de la Organización Europea de Fraseología (EUROPHRAS) y desde el 2008 de la universidad Trier, Alemania. Además, trabajan unos 250 lingüistas honoríficamente en el proyecto que investiga más de 70 lenguas europeas. ${ }^{2} \mathrm{El}$ mapa muestra la distribución del modismo llevarse como el perro y el gato en Europa (Piirainen, 2010, p. 174):

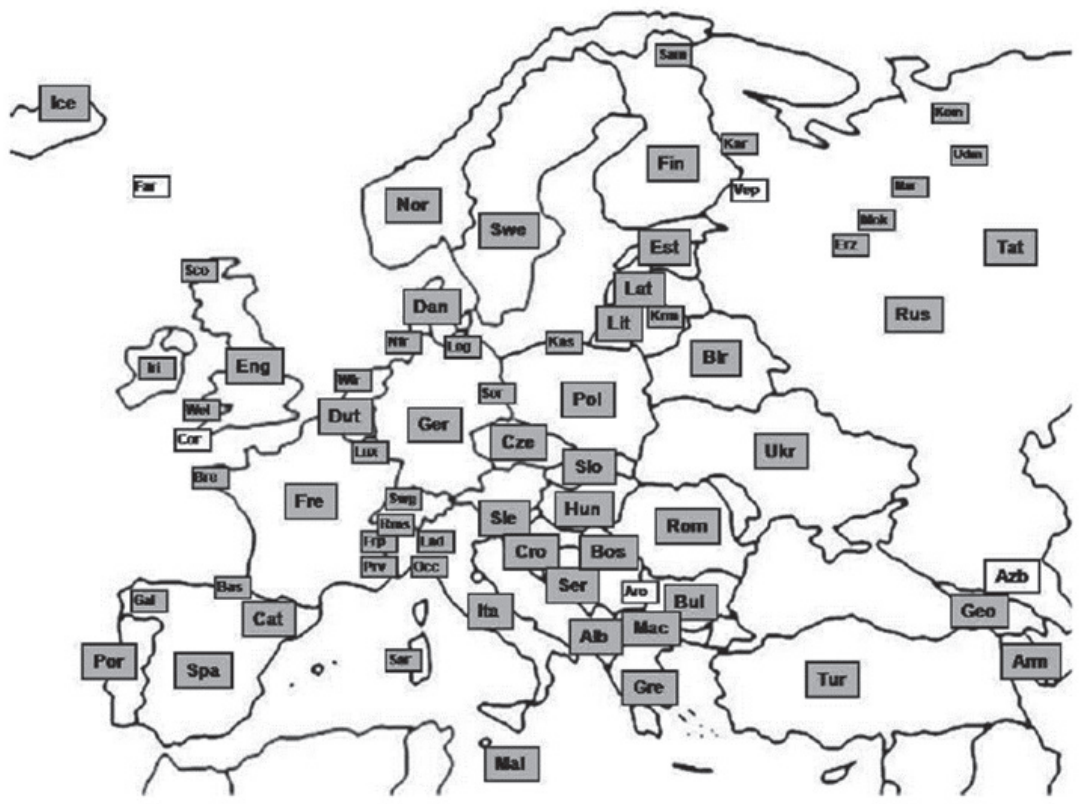

Figura 2. La distribución del modismo llevarse como el perro y el gato en Europa

Otro proyecto que siempre será un work in progress, un proyecto interminable, pero uno que nos da una idea de la complejidad de la fraseología y nos ayuda en la enseñanza de la misma es el del óptimo fraseológico. Ese óptimo contiene los modismos más usados de una lengua y nos sirve para revisar cuáles son las UFs que merecen ser aprendidas durante la adquisición de una segunda lengua. Ya en el campo de los proverbios es difícil acercarse a una suma de los más usados. Según una encuesta que cita Wolfgang Mieder (1992, p. 16), los proverbios considerados más usados en la lengua alemana son los siguientes:

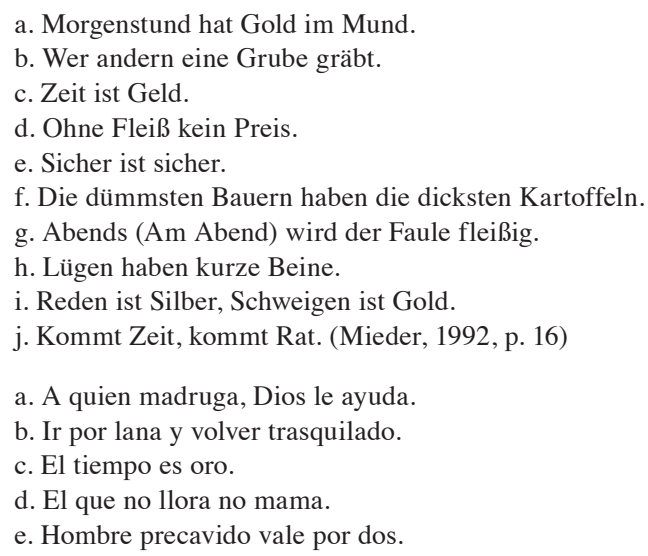


f. "Fortune favors fools".

g. Por la tarde el flojo se transforma en trabajador.

h. Antes se pilla al mentiroso que al cojo.

i. En boca cerrada no entran moscas.

j. El tiempo aclara las cosas. (Mieder, 1992, p. 16, traducción propia)

Como se puede apreciar, en español no existen necesariamente correspondencias entre las UFs de ambas lenguas. Asimismo hay traducciones pero estas no son unidades con los aspectos típicos de la fraseología. Encuestas como esta son dudosas porque cambian mucho según su fuente y además varían con el paso del tiempo. Sin embargo, son un apoyo para contestar la pregunta de cuáles son las paremias que merecen ser enseñadas en el salón de clase.

Otro acercamiento empírico es el proyecto al que se dedicaron Hallsteinsdóttir, Šajánková y Quasthoff en el año 2006. La pregunta de investigación fue si existe un óptimo fraseológico en alemán. Encontraron unos aproximadamente 1112 UFs en una comparación del corpus de textos de la universidad de Leipzig y de los libros de enseñanza. Esos 1112 UFs formaron la base de una encuesta que hicieron después. Según esta encuesta, se hallaron 143 UFs que son los más usados de la lengua alemana, entre otros los siguientes:

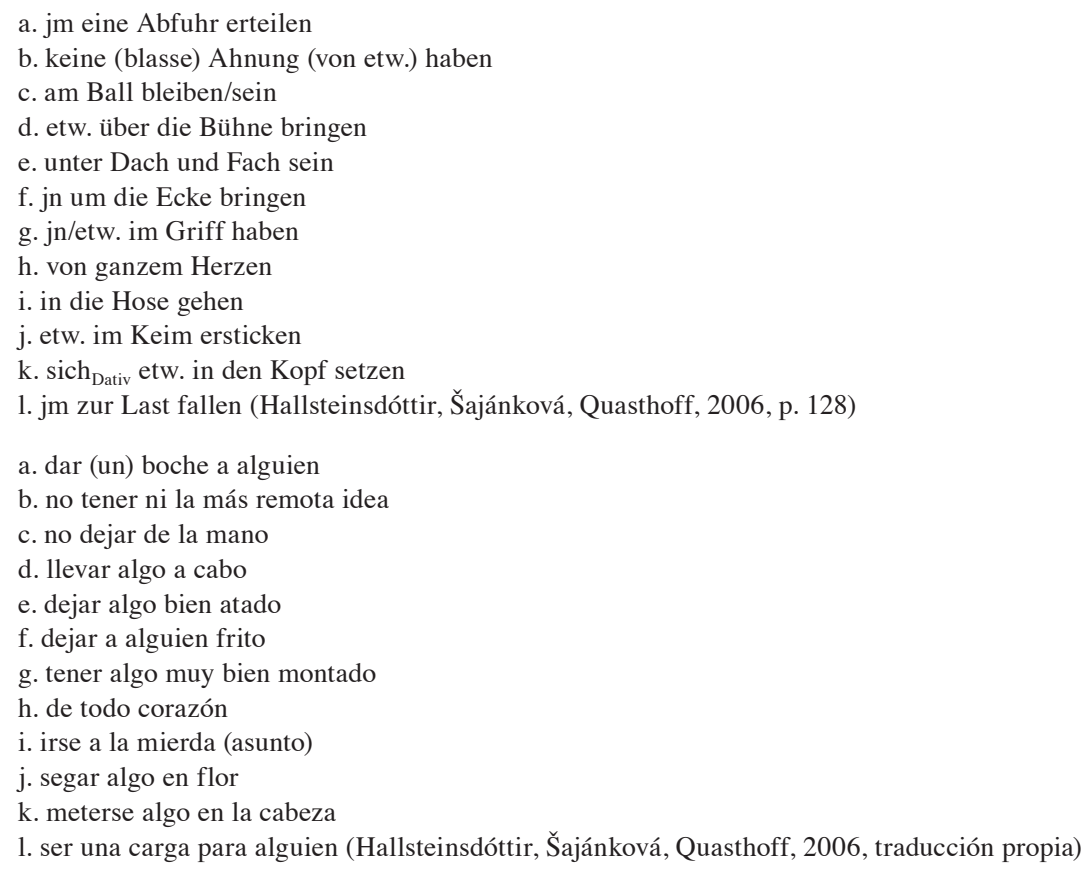

La dificultad de los alumnos de una lengua extranjera se puede ilustrar fácilmente si cada uno tiene en cuenta qué cantidad de UFs sabe reproducir en alguna lengua extranjera una vez alcanzado el nivel B2. A pesar de todo, el trabajo de Hallsteinsdóttir, Šajánková y Quasthoff nos proporciona un posible núcleo, con base empírica de la fraseología como orientación en la enseñanza.

\section{Conclusión}

Los proyectos del óptimo fraseológico y de los "Widespread Idioms" son works in progress: todavía no están listos. Además, el óptimo fraseológico cambiará con cada nueva 
evolución del lenguaje. Es relevante ajustar la enseñanza a través de literatura empleando los resultados de esos proyectos. La investigación de las UFs en literatura nos permite entender la estrategia de los escritores y nos ayuda con el análisis de sus obras. Por último, el uso de la literatura para el aprendizaje de UFs ayuda a los estudiantes en su búsqueda por la palabra correcta, la aguja en el alfiletero que es el lenguaje en general.

\section{Notas}

1. 18 de agosto hasta 6 de octubre del año 2006, Palacio de la Moneda, Santiago de Chile.

2. Véase http://www.widespread-idioms.uni-trier.de (JUN, 2017, párr. 4).

\section{Bibliografía}

Brennan, J. (2007). Prologue. En J. Brennan y A. Taboada (Eds.). How to Survive in the Chilean Jungle. La otra patita. An English Lexicon of Chilean Slang \& Spanish Sayings. (pp. 6-12). Santiago de Chile: Saenz.

Cabrera Infante, G. (1967). Tres tristes tigres. Madrid: Espasa.

Corpas Pastor, G. (1998). Criterios generales de clasificación del universo fraseológico de las lenguas, con ejemplos tomados del español y del inglés. En M. Alvar-Ezquerra y G. Corpas Pastor (Eds.). Diccionarios, Frases, Palabras. (pp. 157-187). Málaga: Servicio de publicaciones de la universidad.

Corpas Pastor, G. (2003). Usos y valores de para nada en un corpus de español peninsular actual. En G. Corpas Pastor (Ed.). Diez años de investigación en fraseología: Análisis sintáctico-semánticos, contrastivos y traductológicos. (pp. 109-123). Madrid: Iberoamericana.

Hallsteinsdóttir, E., Šajánková, M. y Quasthoff, U. (2006). Phraseologisches Optimum für Deutsch als Fremdsprache. Ein Vorschlag auf der Basis von Frequenz- und Gefläufigkeitsuntersuchungen. Linguistik Online. 27. Recuperado de https://bop.unibe. ch/linguistik-online/

Huidobro, V. (2010). Altazor o el viaje en paracaídas. (2 ed.) Madrid: Petrópolis.

Jakobson, R. (1979). Linguistik und Poetik. En E. Holesnstein y T. Schelbert (Eds.). Ausgewählte Aufsätze 1921-1971. (pp. 83-121). Frankfurt am Main: Suhrkamp.

Jandl, E. (1997). Poetische Werke. Por K. Siblewski (Ed.). München: Luchterhand.

Jandl, E. (1969/1971/2001). laut+luise, hosi+anna. Sprechgedichte. [CD] Berlín: Wagenbach.

Lüger, H. H. (1997). Anregungen zur Phraseodidaktik. Beiträge zur Fremdsprachenvermittlung. 32, 69-120.

Mieder, W. (1992). Sprichwort - Wahrwort!? Studien zur Geschichte, Bedeutung und Funktion deutscher Sprichwörter. Frankfurt am Main: Lang.

Parra, N. (1996). Trabajos Prácticos / Hojas de Parra. Santiago de Chile: CESOC.

Parra, N. (2006a). Obras completas \& algo †. Por N. Binns. (Ed.) Barcelona: Galaxia Gutenberg. 
Parra, N. (2006b). Obras Públicas. Santiago de Chile: W.R.S.

Piirainen, E. (2010). Weit verbreitete Idiome in kulturhistorischen Bezügen: zum Verbreitungsmodus einiger Idiomgruppen. En Das Wort. Germanistisches Jahrbuch Russland 2010. (pp. 167-181). Bonn/Moskau: DAAD.

Piirainen, E. (2012). Widespread Idioms in Europe and Beyond. Toward a Lexicon of Common Figurative Units. Nueva York: Lang.

Segura García, B. (1997). Kulturspezifische Phraseologismen in literarischen Texten und ihre Interferenzen beim Übersetzen vom Spanischen ins Deutsche. En A. Sabban (Ed.). Phraseme im Text. Beiträge aus romanistischer Sicht. (pp. 221-236). Bochum: Brockmeyer.

Zuluaga, A. (2006). Sobre las funciones de los fraseologismos en textos literarios. Paremia. 6, 631-640. 